\title{
Secuencias de cultivo alternativas para incrementar el potencial forrajero y productividad del agua
}

\section{Alternative crop sequences for increasing the forage potential and water productivity}

\author{
David Guadalupe Reta Sánchez a*, J. Santos Serrato Corona ${ }^{b}$, Héctor Mario Quiroga Garzaa, \\ Arturo Gaytán Mascorroa , Uriel Figueroa Viramontes ${ }^{a}$
}

\begin{abstract}
RESUMEN
La intensificación de la producción de forraje durante el período otoño-invierno puede incrementar la productividad de los sistemas de producción. El objetivo de este estudio fue determinar el potencial forrajero y productividad del agua (PA) de secuencias de cultivo alternativas con doble cosecha en otoño-invierno. El estudio se realizó durante los ciclos 2012-2013 y 2013-2014 en Matamoros, Coahuila, México. Se compararon las secuencias alternativas canola-triticale-maíz, canola-cebada-maíz, canolacártamo-maíz y canola-triticale-mijo perla con las secuencias convencionales avena-maíz-maíz y avena-sorgo-sorgo. Se determinó la composición química del forraje, los rendimientos de materia seca (MS), nutrientes y PA en la producción de MS, proteína cruda (PC) y energía neta para lactancia $\left(E N_{L}\right)$. Las secuencias alternativas mostraron mayores rendimientos (11.4-53.3 \%) y valores superiores de PA (23.1-58.9 \%) en la producción de PC $(P<0.05)$, además de ahorros en lámina de riego anual de 5.7 a $36.0 \mathrm{~cm}$ al compararse con la secuencia convencional avena-maíz-maíz. Las mejores secuencias alternativas fueron las que incluyeron canola en otoño, cebada o triticale en invierno, y maíz en primavera, debido a su mayor rendimiento y PA en PC ( $P<0.05)$; además, sus valores de PA para MS $\left(1.92-2.05 \mathrm{~kg} \mathrm{~m}^{-3}\right)$ y EN $\left(12.22-13.64 \mathrm{MJ} \mathrm{m}^{-3}\right)$ fueron similares $(P>0.05) 0$ mayores $(P<0.05)$ a los de la secuencia avena-maíz-maíz con 1.65-1.99 $\mathrm{kg} \mathrm{m}^{-3}$ para MS y 11.09-13.72 $\mathrm{MJ} \mathrm{m}^{-3}$ para EN . Los resultados indican que las secuencias alternativas con doble cosecha en otoño-invierno pueden mejorar la eficiencia de producción de forraje.
\end{abstract}

PALABRAS ClAVE: Materia seca, Nutrientes, Composición química, Brassica napus L., Carthamus tinctorius L., Pennisetum glaucum (L.) R. Br.

\section{ABSTRACT}

Intensification of forage production during fall-winter season may improve productivity of cropping systems. The objective of this study was to determine the forage potential and water productivity (WP) of alternative crop sequences with double-crop during fall-winter season. The study was conducted during 2012-2013 y 2013-2014 cycles in Matamoros, Coahuila, México. The alternative crop sequences canola-triticale-corn, canola-barley-corn, canola-safflower-corn and canola-triticale-pearl millet were compared with the conventional crop sequences oat-corn-corn and oat-sorghum-sorghum. Forage chemical composition, dry matter (DM) and nutrient yields, and WP values for DM, crude protein (CP) and net energy for lactation (NEL) production were determined. Alternative crop sequences showed higher yields $(11.4-53.3 \%)$ and superior WP values $(23.1-58.9 \%)$ in $C P$ production $(P<0.05)$, besides they had water savings from 5.7 to $36 \mathrm{~cm}$ as compared with the conventional crop sequence oat-corn-corn. The best alternative sequences were those with the incorporation of canola in fall, barley or triticale in winter, and corn in spring, due to their higher yield and WP in CP $(P<0.05)$. In addition, their WP values for $D M\left(1.92-2.05 \mathrm{~kg} \mathrm{~m}^{-3}\right)$ and $\mathrm{NE}_{\mathrm{L}}\left(12.22-13.64 \mathrm{MJ} \mathrm{m}^{-3}\right)$ were similar $(P>0.05)$ or higher $(P<0.05)$ to those for the sequence oat-corn-corn, with 1.65-1.99 $\mathrm{kg} \mathrm{m}^{-3}$ for $\mathrm{DM}$ and $11.09-13.72 \mathrm{MJ} \mathrm{m}^{-3}$ for $\mathrm{NE}_{\mathrm{L}}$. The results show that alternative crop sequences with double-crop during fall-winter season can improve the efficiency of forage production.

KEY WORDS: Dry matter, Nutrients yields, Chemical composition, Brassica napus L., Carthamus tinctorius L., Pennisetum glaucum (L.) R. Br.

Recibido el 11 de octubre de 2016. Aceptado el 15 de febrero de 2017.

a Campo Experimental La Laguna. Instituto Nacional de Investigaciones Forestales, Agrícolas y Pecuarias. México.

b Facultad de Agricultura y Zootecnia, Universidad Juárez del Estado de Durango. México.

*Autor de correspondencia: dretasan@yahoo.com 


\section{INTRODUCCIÓN}

La producción de leche de bovino en la Comarca Lagunera de México es una de las principales actividades económicas. La producción del forraje utilizado para cubrir las necesidades nutricionales del ganado se realiza bajo irrigación, lo cual constituye una limitante debido a la escasez de agua en la región. El cambio climático global también afecta negativamente la producción de forraje debido al incremento continuo de la temperatura y la ocurrencia de periodos de sequía más prolongados, situación que es altamente probable se agrave en las próximas décadas ${ }^{(1)}$.

En la Comarca Lagunera, las principales secuencias con especies anuales son maíz-maízavena y sorgo-sorgo-avena; de estas secuencias la de mayor productividad es la primera de ellas, con rendimientos de $40 \mathrm{t} \mathrm{ha}^{-1}$ de $\mathrm{MS}, 3,689 \mathrm{~kg} \mathrm{ha}^{-1}$ de $P C$ y de $234,104 \mathrm{MJ} \mathrm{ha}^{-1}$ de $E \mathrm{~N}_{\mathrm{L}}$, con una lámina de riego anual de $2 \mathrm{~m}^{(2)}$. Con estos patrones de cultivo se produce una gran parte del forraje requerido por el ganado lechero en la región; sin embargo, la baja disponibilidad de agua, la salinidad en el suelo, alta temperatura ambiental y un número limitado de cultivos forrajeros ${ }^{(3)}$, condiciona la búsqueda de nuevas estrategias para la producción de forraje con especies forrajeras alternativas, y el desarrollo de nuevos sistemas de producción con potencial para incrementar la productividad del agua (PA), considerando ésta como la producción de MS y nutrientes por $\mathrm{m}^{3}$ de agua aplicada.

Las especies alternativas canola (Brassica napus L.), cártamo (Carthamus tinctorius L.) ${ }^{(3)}$ y mijo perla [Pennisetum glaucum (L.) R. Br] ${ }^{(4)}$, por sus características de precocidad y buena calidad de forraje, junto con especies convencionales como triticale (X Triticosecale Wittmach), cebada (Hordeum vulgare L.) y maíz (Zea mays L.), pueden ser utilizadas para desarrollar sistemas de producción con doble cosecha en otoño-invierno y una en primavera, y así concentrar la producción de forraje en períodos del año donde la temperatura y evapotranspiración son menores. El objetivo de este estudio fue determinar el potencial forrajero $y$ productividad del agua (PA) de secuencias de cultivo alternativas con doble cosecha en otoño-invierno.

\section{MATERIAL Y MÉTODOS}

El estudio se realizó en el Campo Experimental La Laguna del Instituto Nacional de Investigaciones Forestales, Agrícolas y Pecuarias (INIFAP), localizado en Matamoros, Coahuila, México ( $25^{\circ} 32^{\prime} \mathrm{N}, 103^{\circ} 14^{\prime}$ O y $1,150 \mathrm{msnm}$ ), en un suelo de textura arcillosa. El sitio experimental tiene suelos profundos ( $>1.8$ $\mathrm{m})$, con valores de disponibilidad de agua de 150 $\mathrm{mm} \mathrm{m}^{-1(5)}$ y un contenido de $C$ orgánico de $0.75 \%{ }^{(6)}$.

Se evaluaron seis secuencias de cultivos, cuatro de ellas con especies alternativas y dos con las usadas comúnmente, las cuales se consideraron como tratamientos testigo. Las secuencias con especies alternativas fueron: 1) canola-triticale-maíz (Cn-Tcl-Mz); 2) canola-cebada-maíz (Cn-Cb-Mz); 3) canola-cártamo-maíz (Cn-Ctm-Mz); 4) canolatriticale-mijo perla (tres cortes) (Cn-Tcl-MP). Las secuencias testigos fueron: 5) avena-maíz-maíz (Av$\mathrm{Mz}-\mathrm{Mz}$ ); y 6) avena-sorgo-sorgo (Av-Sr-Sr). Se utilizó un diseño experimental de bloques completos al azar con cuatro repeticiones. Las parcelas experimentales fueron de $7 \mathrm{~m}$ de ancho por $6 \mathrm{~m}$ de longitud. La parcela útil en todos los cultivos fue de $15 \mathrm{~m}^{2}$. La preparación del terreno para cada cultivo consistió de un paso de arado a $0.30 \mathrm{~m}$ de profundidad, seguido de doble rastreo y nivelación con escrepa.

La canola se sembró en seco en los ciclos 20122013 y 2013-2014; en el primero la siembra se realizó el 26 de septiembre de 2012 en las secuencias de cultivo 1, 2, 3 y 4 . En el segundo ciclo, la canola se sembró el 25 de septiembre de 2013 en las secuencias 1,2 y 3; mientras que en la secuencia 4, la siembra se realizó el 5 de octubre de 2013. La siembra se efectuó con una sembradora 'Brillion', utilizando $12 \mathrm{~kg} \mathrm{ha}^{-1}$ de semilla del híbrido 'Hyola 401'. A los 15 días después de la siembra (dds) se realizó un aclareo de plantas dejando una densidad de población de 150 plantas $\mathrm{m}^{-2}$. Se aplicó manualmente una fertilización de $200 \mathrm{~kg} \mathrm{~N}^{-1}$ y 60 $\mathrm{kg} \mathrm{ha}{ }^{-1}$ de $\mathrm{P}_{2} \mathrm{O}_{5}$, utilizando sulfato de amonio y fosfato mono amónico. Antes de la siembra se aplicaron $75 \mathrm{~kg} \mathrm{~N}$ ha $^{-1}$ y todo el fósforo, incorporando el fertilizante con un paso de rastra; en el primer riego de auxilio se aplicaron $125 \mathrm{~kg} \mathrm{~N}$ $\mathrm{ha}^{-1}$. 
En canola se aplicó el riego de siembra y un sobre riego seis días después, con 12 y $6 \mathrm{~cm}$ de lámina, respectivamente. Durante el ciclo se dieron dos riegos de auxilio a los 30 y 51 dds, con láminas de $13 \mathrm{~cm}$ cada uno. La cosecha se realizó a los 7172 dds en el primer ciclo; en el segundo se efectuó a los 72 dds en las secuencias 1,2 y 3 , mientras que en la secuencia de cultivo 4 se cosechó a los 89 dds; en todos los casos cuando la canola alcanzó la fase de floración completa (4.4) ${ }^{(7)}$.

La avena, en las secuencias de cultivo 5 y 6 se sembró el 26 de octubre en 2012 y 30 de octubre en 2013 con una sembradora de granos pequeños a una densidad de $140 \mathrm{~kg} \mathrm{ha}^{-1}$. Se utilizó la variedad 'Cuauhtémoc'. El método y dosis de fertilización utilizados fueron iguales a los de la canola. Se aplicó el riego de siembra y un sobre riego $11 \mathrm{dds}$, con 13 y $6 \mathrm{~cm}$ de lámina, respectivamente. Durante el desarrollo del cultivo se aplicaron tres riegos de auxilio a los 40,70 y $92 \mathrm{dds}$, con $13 \mathrm{~cm}$ de lámina cada uno. La cosecha se realizó a los 117 dds, cuando la avena alcanzó la fase de espigado e inicio de formación del grano.

En los ciclos 2012-2013 y 2013-2014, la siembra de invierno se realizó en seco con una sembradora para granos pequeños en las parcelas donde se cosechó la canola. Las especies sembradas fueron triticale (secuencias 1 y 4 ), cebada (secuencia 2) y cártamo (secuencia 3) el 18 y 20 de diciembre de 2012 y 2013, respectivamente. Se utilizó la variedad 'Río Nazas' de triticale, 'San Marcos' de cebada y 'Gila' de cártamo. La densidad de siembra en triticale fue de $140 \mathrm{~kg} \mathrm{ha}^{-1}$, en cebada de $120 \mathrm{~kg} \mathrm{ha}^{-1} \mathrm{y}$ en cártamo de $90 \mathrm{~kg} \mathrm{ha}^{-1}$. En cártamo se realizó un aclareo de plantas a los 25 dds, dejando una densidad de población de 100 plantas $\mathrm{m}^{-2}$. En los tres cultivos, los métodos y dosis de fertilización utilizados fueron iguales a los mencionados para canola y avena.

En triticale y cebada se aplicaron tres riegos de auxilio a los 46, 69 y 91 dds; en cártamo se aplicaron dos riegos de auxilio a los 46 y 69 dds. La cosecha del triticale se realizó cuando el grano estaba en estado lechoso a los 109 dds en la secuencia de cultivo 1, mientras que en la secuencia de cultivo 4 se realizó a los 91 dds en la misma etapa del desarrollo. La cebada se cosechó en la fase de grano lechoso-masoso a los 106 dds, en tanto que el cártamo se cosechó en la fase de inicio de botón floral, a los 91 dds.

En la estación de primavera, el maíz y el sorgo en los testigos (secuencias de cultivo 5 y 6) se sembraron en suelo húmedo el 26 de marzo de 2013 y 2014, utilizando el híbrido de maíz 'Garst 8285' y el sorgo 'Silo Miel'. En maíz se realizó un aclareo de plantas a los $20 \mathrm{dds}$, dejando una densidad de población de 100 mil plantas ha ${ }^{-1}\left(37 \mathrm{~kg} \mathrm{ha}^{-1}\right)$, mientras que en sorgo se utilizaron $15 \mathrm{~kg}$ de semilla $\mathrm{ha}^{-1}$. En las secuencias alternativas se sembraron maíz y mijo perla en suelo húmedo el 29 de abril de 2013. En 2014 el maíz en las secuencias de cultivo 1,2 y 3 se sembró el 14 de abril, mientras que el mijo perla en la secuencia de cultivo 4 se estableció el 6 de mayo, utilizando la variedad 'Tifleaf 3'. En la siembra de ambos cultivos, tanto en los testigos como en las secuencias alternativas, se utilizó una distancia entre surcos de $0.76 \mathrm{~m}$. La densidad de población del maíz en las secuencias alternativas fue igual a la utilizada en el maíz del testigo (secuencia $5)$, mientras que en mijo perla se utilizó una densidad de siembra de $20 \mathrm{~kg} \mathrm{ha}^{-1}$.

En los dos ciclos de evaluación el maíz y sorgo se fertilizaron con $250 \mathrm{~kg} \mathrm{~N}$ y $80 \mathrm{~kg} \mathrm{P}_{2} \mathrm{O}_{5} \mathrm{ha}^{-1}$, utilizando sulfato de amonio y fosfato mono amónico como fuentes. El total de fósforo se aplicó en la siembra; mientras que el $\mathrm{N}$ se aplicó el $25 \%$ en la siembra, el $50 \%$ en el primer riego de auxilio y el $25 \%$ en el segundo. En mijo perla se aplicó en la siembra $80 \mathrm{~kg} \mathrm{~N} \mathrm{ha}^{-1}$ y $100 \mathrm{~kg} \mathrm{P}_{2} \mathrm{O}_{5}$, posteriormente se fertilizó con $90 \mathrm{~kg} \mathrm{~N} \mathrm{ha}^{-1}$ en el primer riego de auxilio y $80 \mathrm{~kg} \mathrm{~N} \mathrm{ha}^{-1}$ en el riego de auxilio después del primer corte.

El número y calendario de riegos fue el mismo en los dos ciclos de evaluación. Se aplicaron el riego de presiembra y cuatro de auxilio en maíz y sorgo, a los 35, 51, 68 y 85 dds. En mijo perla fueron cinco riegos para los tres cortes, dos para el primero y el segundo, y uno para el tercero. Los riegos se dieron a los 39, 60, 80, 101 y 119 dds. La cosecha de maíz en todos los tratamientos se realizó en la etapa de un tercio de la línea de leche del grano a los 101 dds; mientras que en sorgo se efectuó en grano lechoso. En mijo perla los cortes se realizaron a los 79, 115 y 144 dds en la etapa de antesis. 
En la estación de verano, en los testigos (secuencias 5 y 6 ) se establecieron el maíz y el sorgo el 15 y 18 de julio de 2013 y 2014 , respectivamente. En maíz se utilizó el híbrido 'Garst 8285 ' con una densidad de siembra de 100 mil semillas ha-1. La siembra se realizó en suelo seco, aplicando el riego de siembra el mismo día. A los 15 dds se realizó un aclareo de plantas, dejando una densidad de población de 90 mil plantas ha-1 ${ }^{-1}$ ya que existe una menor respuesta a la densidad de población en verano. En sorgo se desarrolló el rebrote del mismo establecido en primavera. El distanciamiento entre surcos, dosis y método de fertilización en maíz y sorgo fueron iguales a los utilizados en la estación de primavera. En ambos cultivos se aplicaron cuatro riegos de auxilio a los 24, 42, 58 y 77 dds con una lámina de riego de $13 \mathrm{~cm}$ cada uno. La cosecha se realizó a los 99 dds cuando el grano alcanzó la etapa de un tercio de la línea de leche, y el sorgo durante la etapa de grano lechoso.

En los cultivos de avena, canola, cebada, triticale y cártamo se realizó el control del pulgón verde (Acyrthosiphon pisum Harris) con Dimetoato $40 \mathrm{EC} \cap$ (Dimetoato; 0,0-dimetil S-metilcarbamoilmetil fosforoditioato) a una dosis de $1.0 \mathrm{~L} \mathrm{ha}^{-1}$; en cada ciclo, se realizó una aplicación a los 50-55 dds. El control de plagas en maíz, sorgo y mijo perla se realizó con dos aplicaciones de insecticidas en cada ciclo de crecimiento; a los 20 dds se aplicó Lorsban $480 \AA$ (Clorpirifos etil; 0,0-dietil 0-3,5,6- trichloropyridin-2-il fosforotioato) en dosis de $1 \mathrm{~L} \mathrm{ha}^{-1}$ para el control de gusano cogollero (Spodoptera frugiperda). Posteriormente, a los 60 dds se aplicó agromectin 1.8 C.E. ${ }^{R}$ (Abamectina; Avermectina B1aC48H72O14 + Avermectina B1bC47H70014) en dosis de $1.0 \mathrm{~L} \mathrm{ha}^{-1}$ para el control de araña roja (Tetranychus spp). En todos los cultivos establecidos en las secuencias de cultivo la maleza se controló en forma manual y con azadón.

En la cosecha se determinó en cada cultivo y secuencia de cultivo el rendimiento de forraje fresco, y en una muestra por parcela $\left(1 \mathrm{~m}^{2}\right)$ se obtuvo el porcentaje de materia seca (MS) y la composición química del forraje. Estas plantas se secaron en una estufa de aire forzado a $60^{\circ} \mathrm{C}$ hasta peso constante. El rendimiento de $M S$, se estimó mediante el producto del rendimiento de forraje fresco por el porcentaje de MS. En composición química del forraje, se determinó fibra detergente ácido (FDA) y fibra detergente neutro (FDN) ${ }^{(8)} ; \mathrm{N}$ con el método Kjeldahl( $^{(9)}$ y el contenido de $\mathrm{EN}_{\mathrm{L}}$ de acuerdo con las metodologías del Consejo Nacional de Investigación $^{(10)}$. Con estos datos se calcularon los rendimientos de $\mathrm{PC}$ y $\mathrm{EN}_{\mathrm{L}} \mathrm{ha}^{-1}$, multiplicando el contenido de estos nutrientes en el forraje con el rendimiento de MS en cada cultivo y secuencia.

Se midió el volumen de agua aplicada en cada secuencia de cultivo, aforando el caudal de agua en las compuertas de los tubos instalados para el riego y considerando el tiempo de riego en cada parcela experimental. La productividad del agua (PA) en las secuencias se estimó con el cociente del rendimiento de MS, PC y EN $N_{L}$ entre el volumen total del agua aplicada.

Se hicieron análisis de varianza para los datos de rendimiento, productividad del agua en la producción de materia seca y nutrientes de las secuencias de cultivo $(P<0.05)$. El análisis estadístico de todas las variables es presentado por ciclo, ya que las condiciones climáticas en los dos ciclos del estudio fueron diferentes. Para comparar las medias se utilizó la prueba de la diferencia mínima significativa protegida de Fisher $(P<0.05)$. Los datos se analizaron con el programa estadístico SAS ${ }^{(11)}$.

\section{RESULTADOS Y DISCUSIÓN}

\section{Características del clima durante el estudio}

Durante las estaciones de otoño e invierno se registraron temperaturas máximas y mínimas menores con relación a las estaciones de primavera y verano, en las cuales se alcanzaron las mayores temperaturas durante el ciclo, además de la mayor evaporación potencial (Cuadro 1). La comparación entre los ciclos 2012-2013 y 2013-2014 indica que las temperaturas medias de máximas y mínimas en primavera y verano, respectivamente, fueron mayores en el primer ciclo. La precipitación fue mayor en el segundo ciclo $(337.8 \mathrm{~mm})$ que en el primero $(254.6 \mathrm{~mm})$, además mostró una mejor distribución, ya que las lluvias ocurrieron en las cuatro estaciones del año. 


\section{Cíclo de crecimiento y composición química del forraje}

La duración del ciclo de crecimiento y la composición química del forraje de las especies incluidas en las secuencias de cultivo se presentan en el Cuadro 2. Las especies de otoño-invierno canola, cártamo, cebada y triticale fueron más precoces (72-106 días) que el testigo avena (117 días). En composición química la avena como especie forrajera convencional producida durante las estaciones de otoño e invierno presentó concentraciones de $115.2 \mathrm{~g} \mathrm{~kg}^{-1}$ de PC, $584.1 \mathrm{~g} \mathrm{~kg}^{-1}$ de FDN y $5.70 \mathrm{MJ} \mathrm{kg}^{-1} \mathrm{MS}$ de EN. Al comparar la avena con el forraje de los cultivos alternativos de otoño-invierno, canola y cártamo, estos últimos presentaron mayores contenidos de PC (171.3 a $184.2 \mathrm{~g} \mathrm{~kg}^{-1}$ ) y menores concentraciones de FDN (407.1 a $493.4 \mathrm{~g} \mathrm{~kg}^{-1}$ ). El contenido de $\mathrm{EN}_{\mathrm{L}}$ en canola

Cuadro 1. Período de crecimiento (Pcrec) por estación y factores climáticos durante el desarrollo de las especies establecidas en las secuencias de cultivo evaluadas en los ciclos 2012-2013 y 2013-2014

\begin{tabular}{|c|c|c|c|c|c|c|c|}
\hline & \multirow{2}{*}{ Fecha de siembra } & \multirow{2}{*}{ Cosecha } & \multirow{2}{*}{ Pcrec (días) } & \multicolumn{2}{|c|}{ Temperatura media $\left({ }^{\circ} \mathrm{C}\right)$} & \multirow{2}{*}{$\begin{array}{c}\text { Precipitación } \\
\text { acumulada }(\mathrm{mm})\end{array}$} & \multirow{2}{*}{$\begin{array}{c}\text { Evaporación } \\
\text { potencial (mm) }\end{array}$} \\
\hline & & & & Máxima & Mínima & & \\
\hline \multicolumn{8}{|c|}{ 2012-2013 } \\
\hline Otoño & 26 sept & 25 ene & 121 & 25.9 & 9.6 & 28.8 & 551.9 \\
\hline Invierno & $18 \mathrm{dic}$ & $8 \mathrm{abr}$ & 111 & 25.4 & 7.2 & 0 & 636.1 \\
\hline Primavera & 27 mar & 9 jul & 104 & 33.8 & 17.5 & 29.6 & 657.3 \\
\hline Verano & $10 \mathrm{jul}$ & 21 oct & 103 & 32.2 & 19.7 & 196.2 & 557.5 \\
\hline \multicolumn{8}{|c|}{ 2013-2014 } \\
\hline Otoño & 25 sept & 24 ene & 121 & 24.5 & 9.7 & 148.2 & 574.3 \\
\hline Invierno & $20 \mathrm{dic}$ & $8 \mathrm{abr}$ & 109 & 25.4 & 7.9 & 37.8 & 608.2 \\
\hline Primavera & 26 mar & 11 jul & 107 & 33.0 & 18.3 & 73.6 & 646.2 \\
\hline Verano & 16 jul & 24 oct & 100 & 32.6 & 19.4 & 78.2 & 545.8 \\
\hline
\end{tabular}

Cuadro 2. Período de crecimiento (Pcrec) y parámetros de la composición química del forraje de cada especie incorporada en las secuencias de cultivo evaluadas en los ciclos 2012-2013 y 2013-2014

\begin{tabular}{|c|c|c|c|c|c|}
\hline \multirow{2}{*}{ Especies } & \multirow{2}{*}{ Pcrec (días) } & \multicolumn{4}{|c|}{ Parámetros de la composición química } \\
\hline & & $P C\left(\mathrm{~g} \mathrm{~kg}^{-1}\right)$ & FDN $\left(\mathrm{g} \mathrm{kg}^{-1}\right)$ & FDA $\left(\mathrm{g} \mathrm{kg}^{-1}\right)$ & $\mathrm{EN}$ ( $\left.\mathrm{MJ} \mathrm{kg}^{-1} \mathrm{MS}\right)$ \\
\hline Avena & 117 & $115.2 \ddagger$ & 584.1 & 356.8 & 5.70 \\
\hline Canola & 72 & 184.2 & 407.1 & 340.0 & 5.90 \\
\hline Cebada & 106 & 96.7 & 536.7 & 343.8 & 5.83 \\
\hline Triticale & 110 & 115.4 & 595.8 & 360.4 & 5.67 \\
\hline Cártamo & 95 & 171.3 & 493.4 & 399.8 & 5.24 \\
\hline Maíz primavera & 101 & 68.3 & 465.5 & 233.7 & 7.02 \\
\hline Maíz verano & 99 & 69.4 & 415.1 & 209.5 & 7.31 \\
\hline Sorgo primavera & 106 & 59.0 & 552.1 & 354.3 & 5.74 \\
\hline Sorgo verano & 100 & 52.4 & 591.9 & 408.2 & 5.13 \\
\hline Mijo Perla & 140 & 127.7 & 617.1 & 376.2 & 5.48 \\
\hline
\end{tabular}

$\mathrm{PC}=$ proteína cruda; $\mathrm{FDN}=$ fibra detergente neutro; $\mathrm{FDA}=$ fibra detergente ácido; $\mathrm{EN}=$ energía neta para lactancia.

$\ddagger \ddagger$ Valores promedio de las parcelas con cada especie evaluada en los ciclos 2012-2013 y 2013-2014. 
(5.90 MJ kg-1 MS) fue similar al de la avena; mientras que en cártamo la concentración (5.24 MJ kg-1 MS) fue menor.

En la estación de invierno, el triticale cosechado en grano lechoso presentó una composición química similar a la obtenida en avena; mientras que en cebada cosechada en la etapa de grano lechosomasoso se obtuvieron menores contenidos de PC $\left(96.7 \mathrm{~g} \mathrm{~kg}^{-1}\right)$, de FDN $\left(536.7 \mathrm{~g} \mathrm{~kg}^{-1}\right)$ y de FDA $(343.8$ $\mathrm{g} \mathrm{kg}^{-1}$ ) y un mayor contenido de $\mathrm{EN}_{\mathrm{L}}\left(5.83 \mathrm{MJ} \mathrm{kg}^{-1}\right.$ MS) que en avena (Cuadro 2).

En primavera-verano, el maíz registró mayores contenidos de $\mathrm{EN}_{\mathrm{L}}\left(7.02\right.$ a $\left.7.31 \mathrm{MJ} \mathrm{kg}^{-1} \mathrm{MS}\right)$ y PC $\left(68.3\right.$ a $\left.69.4 \mathrm{~g} \mathrm{~kg}^{-1}\right)$, además de menores valores de FDN (415.1 y $465.5 \mathrm{~g} \mathrm{~kg}^{-1}$ ) que el sorgo, el cual registró concentraciones de 5.13 a $5.74 \mathrm{MJ} \mathrm{kg}^{-1} \mathrm{MS}$ en $\mathrm{EN}_{\mathrm{L}}$, de 52.4 a $59.0 \mathrm{~g} \mathrm{~kg}^{-1}$ en PC y de 552.1 a $591.9 \mathrm{~g} \mathrm{~kg}^{-1}$ en FDN. El mijo perla cosechado en la etapa de antesis presentó una concentración de PC mayor $\left(127.7 \mathrm{~g} \mathrm{~kg}^{-1}\right)$ a las observadas en maíz y sorgo, pero con un mayor contenido de FDN (617.1 $\mathrm{g} \mathrm{kg}^{-1}$ ) y menor valor de $\mathrm{EN}_{\mathrm{L}}\left(5.48 \mathrm{MJ} \mathrm{kg}^{-1} \mathrm{MS}\right)$ (Cuadro 2).

Al comparar la composición química del forraje de canola y cártamo con el de la avena, se obtuvieron resultados similares a los observados en estudios previos realizados en la región. En estos trabajos se observó consistentemente una mejor composición química del forraje de canola, con una mayor concentración de PC $\left(159.3\right.$ a $\left.240.3 \mathrm{~g} \mathrm{~kg}^{-1}\right)$ y menor contenido de FDN $\left(390.1 \mathrm{a} 466.0 \mathrm{~g} \mathrm{~kg}^{-1}\right)$ que el obtenido en el forraje de avena, la cual presentó valores de PC de 99.0 a $134.0 \mathrm{~g} \mathrm{~kg}^{-1}$ y de FDN de 620.0 a $687.4 \mathrm{~g} \mathrm{~kg}^{-1}(2,12,13)$.

En otros estudios también se han encontrado ventajas en la composición química del forraje de cártamo respecto a la obtenida en avena, con mayor contenido de PC (175.2 a $\left.185 \mathrm{~g} \mathrm{~kg}^{-1}\right)$ y menor concentración de FDN (393.0 a $\left.455 \mathrm{~g} \mathrm{~kg}^{-1}\right)$. Sin embargo, también se han detectado desventajas en el contenido de $\mathrm{EN}_{\mathrm{L}}$, ya que en cártamo cosechado en inicio de yema floral, se han observado valores menores (4.52 a $5.82 \mathrm{MJ} \mathrm{kg}^{-1} \mathrm{MS}$ ) a los de la avena ${ }^{(3,14,15)}$.

En trabajos realizados sobre la utilización de canola y cártamo en la alimentación del ganado bovino lechero, se ha encontrado que su forraje no afectó la producción de leche ni su composición química ${ }^{(16,17)}$. En el presente estudio, los resultados de la composición química de ambos forrajes sugieren que son de buena calidad; sin embargo, es necesario realizar estudios adicionales de consumo y respuesta del ganado lechero para corroborarlo.

En la estación de invierno, el contenido de PC en los forrajes de triticale $\left(115.4 \mathrm{~g} \mathrm{~kg}^{-1}\right)$ y cebada $(96.7 \mathrm{~g})$, fue similar a otro estudio realizado en la región ${ }^{(18)}, 107.0 \mathrm{~g}$ en triticale y $104.5 \mathrm{~g}$ en cebada, ambas especies cosechadas en la fase de grano lechoso masoso. También comparados con el estudio antes citado, el valor de FDN en el presente estudio en triticale $\left(595.8 \mathrm{~g} \mathrm{~kg}^{-1}\right)$ fue similar (619.9 $\mathrm{g})$; mientras que en cebada, el contenido de FDN fue mayor $(655.4 \mathrm{~g})$ al obtenido en el presente estudio $(536.7 \mathrm{~g})$.

Las características de composición química del forraje en las especies convencionales maíz y sorgo presentaron valores similares o mejores (Cuadro 2) que los encontrados en estudios previos en la región ${ }^{(2,13)}$. Los contenidos de PC fueron bajos, similares a los reportados previamente, de 62.7 a $85.0 \mathrm{~g} \mathrm{~kg}^{-1}$ en maíz y de 52.6 a $65.3 \mathrm{~g}$ en sorgo. En FDN, las concentraciones fueron menores a las obtenidas anteriormente en maíz (488.0 a $649.2 \mathrm{~g}$ $\mathrm{kg}^{-1}$ ) y sorgo (641.3 a $708.4 \mathrm{~g}$ ), lo cual indica una mejor composición química del forraje en el presente estudio.

\section{Ciclo de crecimiento y requerimientos de agua en secuencias de cultivo}

Como resultado de la mayor precocidad de las especies alternativas de otoño-invierno, el ciclo de crecimiento de tres de las nuevas secuencias (268282 días) fue más corto que el de los testigos (319324 días); la excepción fue la secuencia que incluyó mijo perla, que tuvo un mayor periodo de crecimiento que los testigos. Además, debido a que dos de las especies en las secuencias alternativas se desarrollaron durante otoño e invierno, las temperaturas medias y los valores de evaporación potencial durante el ciclo, además de las láminas de 
riego requeridas por estas secuencias, fueron menores respecto a los testigos (Cuadro 3).

Las secuencias alternativas, con doble cosecha en otoño-invierno y maíz en primavera, presentaron un ahorro de agua anual entre 23.8 y $29.3 \mathrm{~cm}$ el primer ciclo, y entre 24.0 y $36.0 \mathrm{~cm}$ en el segundo, debido al menor requerimiento de agua de los cultivos alternativos de otoño-invierno. En la secuencia $\mathrm{Cn}-\mathrm{Tcl}-\mathrm{Mp}$ se obtuvo un menor ahorro de agua (5.7 a $12.8 \mathrm{~cm}$ ) debido a los mayores requerimientos de agua del mijo perla con los tres cortes durante las estaciones de primavera y verano (Cuadro 3).

\section{Rendimientos de secuencias de cultivo}

En el Cuadro 4 se presentan los rendimientos de MS, PC y EN $N_{L}$ obtenidos en las secuencias de cultivo evaluadas. Entre las secuencias tradicionales,

Cuadro 3. Período de crecimiento (Pcrec), temperatura media (Tmed), evaporación del tanque evaporímetro (Ep) y lámina de riego aplicada (LR) en seis secuencias de cultivo evaluadas durante los ciclos 2012-2013 y 2013-2014

\begin{tabular}{lccccccc}
\hline \multirow{2}{*}{ Secuencias de cultivo } & \multirow{2}{*}{ Ciclo } & \multicolumn{2}{c}{ Fechas de } & \multirow{2}{*}{$\begin{array}{c}\text { Pcrec } \\
\text { (dias })\end{array}$} & $\begin{array}{c}\text { Tmed } \\
\left({ }^{\circ} \mathrm{C}\right)\end{array}$ & $\begin{array}{c}\text { Ep } \\
(\mathrm{cm})\end{array}$ & $\begin{array}{c}\text { LR } \\
(\mathrm{cm})\end{array}$ \\
\cline { 2 - 4 } Av-Mz-Mz & $2012-2013$ & $26 / 10 / 2012$ & $21 / 10 / 2013$ & 319 & 22.3 & 178.1 & 204.8 \\
& $2013-2014$ & $30 / 10 / 2013$ & $24 / 10 / 2014$ & 322 & 22.1 & 176.7 & 204.2 \\
Av-Sr-Sr & $2012-2013$ & $26 / 10 / 2012$ & $21 / 10 / 2013$ & 324 & 22.4 & 178.1 & 200.5 \\
& $2013-2014$ & $30 / 10 / 2013$ & $17 / 10 / 2014$ & 321 & 22.1 & 172.9 & 199.5 \\
Cn-Tcl-Mz & $2012-2013$ & $26 / 09 / 2012$ & $05 / 08 / 2013$ & 281 & 21.3 & 158.5 & 179.2 \\
& $2013-2014$ & $25 / 09 / 2013$ & $24 / 07 / 2014$ & 282 & 21.1 & 155.1 & 179.5 \\
Cn-Cb-Mz & $2012-2013$ & $26 / 09 / 2012$ & $05 / 08 / 2013$ & 276 & 21.3 & 158.5 & 181.0 \\
& $2013-2014$ & $25 / 09 / 2013$ & $24 / 07 / 2014$ & 279 & 21.1 & 153.1 & 180.2 \\
Cn-Ctm-Mz & $2012-2013$ & $26 / 09 / 2012$ & $05 / 08 / 2013$ & 269 & 21.2 & 158.5 & 175.5 \\
& $2013-2014$ & $25 / 09 / 2012$ & $15 / 07 / 2014$ & 268 & 20.8 & 145.8 & 168.2 \\
Cn-Tcl-Mp & $2012-2013$ & $26 / 09 / 2012$ & $20 / 09 / 2013$ & 327 & 22.0 & 183.6 & 192.0 \\
& $2013-2014$ & $05 / 10 / 2013$ & $19 / 09 / 2014$ & 334 & 21.2 & 182.1 & 198.5 \\
\hline
\end{tabular}

$\mathrm{Av}=$ avena: $\mathrm{Mz}=$ maiz: $\mathrm{Sr}=$ sorao: $\mathrm{Cn}=$ canola: $\mathrm{Tcl}=$ triticale: $\mathrm{Cb}=$ cebada: $\mathrm{Ctm}=$ cártamo: $\mathrm{Mn}=$ miio perla.

Cuadro 4. Rendimientos de materia seca (MS), proteína cruda (PC) y energía neta para lactancia ( $\left.E N_{\mathrm{L}}\right)$ en seis secuencias de cultivo evaluadas durante los ciclos 2012-2013 y 2013-2014

\begin{tabular}{lcccccc}
\hline & \multicolumn{6}{c}{ Rendimientos } \\
\cline { 2 - 7 } Secuencias de cultivo & \multicolumn{2}{c}{ MS $\left(\mathrm{kg} \mathrm{ha}^{-1}\right)$} & \multicolumn{2}{c}{ PC $\left(\mathrm{kg} \mathrm{ha}^{-1}\right)$} & \multicolumn{2}{c}{ ENL (MJ ha-1) } \\
& $2012-2013$ & $2013-2014$ & $2012-2013$ & $2013-2014$ & $2012-2013$ & $2013-2014$ \\
\hline Av-Mz-Mz & $35,969 \mathrm{bc}$ & $42,814 \mathrm{a}$ & $2,945 \mathrm{~d}$ & $3,274 \mathrm{~d}$ & $242,107 \mathrm{ab}$ & $295,524 \mathrm{a}$ \\
Av-Sr-Sr & $44,070 \mathrm{a}$ & $39,220 \mathrm{~b}$ & $3,059 \mathrm{~d}$ & $2,507 \mathrm{e}$ & $244,099 \mathrm{ab}$ & $215,848 \mathrm{c}$ \\
Cn-Tcl-Mz & $34,853 \mathrm{bc}$ & $38,841 \mathrm{~b}$ & $3,574 \mathrm{c}$ & $3,993 \mathrm{~b}$ & $221,446 \mathrm{bc}$ & $253,488 \mathrm{~b}$ \\
Cn-Cb-Mz & $37,608 \mathrm{~b}$ & $38,132 \mathrm{~b}$ & $3,740 \mathrm{bc}$ & $3,646 \mathrm{c}$ & $249,521 \mathrm{a}$ & $245,128 \mathrm{~b}$ \\
Cn-Ctm-Mz & $33,790 \mathrm{c}$ & $34,577 \mathrm{c}$ & $3,870 \mathrm{~b}$ & $3,937 \mathrm{~b}$ & $210,895 \mathrm{c}$ & $226,371 \mathrm{c}$ \\
Cn-Tcl-Mp & $34,077 \mathrm{c}$ & $31,660 \mathrm{~d}$ & $4,516 \mathrm{a}$ & $4,443 \mathrm{a}$ & $183,422 \mathrm{~d}$ & $184,456 \mathrm{~d}$ \\
\hline
\end{tabular}

$\mathrm{Av}=$ avena; $\mathrm{Mz}=$ maíz; $\mathrm{Sr}=$ sorgo; $\mathrm{Cn}=$ canola; $\mathrm{Tcl}=$ triticale; $\mathrm{Cb}=$ cebada; $\mathrm{Ctm}=$ cártamo; $\mathrm{Mp}=$ mijo perla.

abcd Medias con la misma letra no son estadísticamente diferentes (DMS 0.05). 
Av-Mz-Mz registró los mejores rendimientos de MS y nutrientes $(P<0.05)$. El primer ciclo, la secuencia tradicional Av-Sr-Sr obtuvo el mayor rendimiento de MS (44,070 kg ha-1) $(P<0.05)$, sin embargo, su rendimiento de PC $\left(3,059 \mathrm{~kg} \mathrm{ha}^{-1}\right)$ y EN $(244,099 \mathrm{MJ}$ $\left.\mathrm{ha}^{-1}\right)$ fueron similares $(P>0.05)$ a la secuencia Av$\mathrm{Mz}-\mathrm{Mz}$, que produjo rendimientos de $35,969 \mathrm{~kg}$, $2,945 \mathrm{~kg}$ y $242,107 \mathrm{MJ}^{\mathrm{ha}}{ }^{-1}$ de MS, PC y EN $\mathrm{L}_{\mathrm{L}}$ respectivamente. En el segundo ciclo, los rendimientos de $M S(42,814 \mathrm{~kg}), \mathrm{PC}(3,274 \mathrm{~kg})$ y $\mathrm{EN}_{\mathrm{L}}$ (295,524 MJ ha-1) en la secuencia Av-Mz-Mz fueron superiores $(P<0.05)$ a los obtenidos por Av-Sr-Sr, debido a un menor rendimiento de MS del sorgo en el verano $(15,070 \mathrm{~kg})$ respecto al obtenido en la misma estación del primer ciclo $(21,803 \mathrm{~kg})$. Además, el rendimiento de MS del maíz en primavera $(20,145 \mathrm{~kg})$ fue mayor al obtenido en la estación de primavera del ciclo anterior $(15,101 \mathrm{~kg})$.

La fluctuación entre ciclos en el rendimiento de MS de maíz y sorgo modificó la comparación del potencial forrajero de las secuencias alternativas con el potencial de las secuencias convencionales, principalmente en la producción de MS y energía. En la producción de PC, las secuencias alternativas mostraron ventajas $(P<0.05)$ en los dos ciclos del estudio (Cuadro 4), debido principalmente a la mayor concentración de este nutriente en las especies alternativas en relación a las convencionales.

Los mejores resultados en rendimientos de MS y nutrientes se obtuvieron en aquellas secuencias que incluyeron cebada y triticale en invierno, las cuales obtuvieron en relación a $\mathrm{Av}-\mathrm{Mz}-\mathrm{Mz}$, rendimientos de
MS, PC y ENL de 89.1 a $90.7 \%, 111.4$ a $127.0 \%$ y 82.9 a $103 \%$, respectivamente. Esta respuesta en ambas secuencias alternativas se debió principalmente a los altos rendimientos de MS obtenidos por triticale y cebada en invierno $(9,127$ - 11,851 kg), además de que los rendimientos de MS de canola en otoño $(6,709-7,163 \mathrm{~kg})$ no fueron muy variables durante los dos ciclos del estudio.

Las secuencias de cultivo $\mathrm{Cn}-\mathrm{Ctm}-\mathrm{Mz}$ y $\mathrm{Cn}-\mathrm{Tcl}-$ Mp fueron sobresalientes en la producción de PC $\left(3,937-4,443 \mathrm{~kg} \mathrm{ha}^{-1}\right)$, pero disminuyeron sus rendimientos de 19.2 a $26.0 \%$, y 23.4 a $37.6 \%$ en la producción de $M S$ y $E N_{L}$, respectivamente $(P<0.05)$; esto debido a los menores rendimientos de MS del cártamo $(7,033$ a 7,760 kg) en invierno y mijo perla $(15,106$ a $16,555 \mathrm{~kg})$ en primaveraverano, además del menor contenido de $\mathrm{EN}_{\mathrm{L}}$ de ambos cultivos, respecto a los cereales de grano pequeño en invierno, y del maíz en primaveraverano (Cuadro 2).

\section{Productividad del agua}

La PA en las secuencias alternativas respecto a la obtenida en las secuencias convencionales Av-Mz$\mathrm{Mz}$ y Av-Sr-Sr fue variable en los dos ciclos del estudio. En el primer ciclo la PA para MS, PC y EN $\mathrm{EN}_{\mathrm{L}}$ en las secuencias alternativas con doble cosecha en otoñoinvierno y una en primavera, fue igual ( $P>0.05)$ o superior $(P<0.05)$ a la de los testigos debido a un menor rendimiento de MS en el maíz de la secuencia tradicional, obteniendo la mayor ventaja en la producción de PC (37.8 - 61.5 \%) (Cuadro 5).

Cuadro 5. Productividad del agua (PA) en la producción de materia seca (MS), proteína cruda (PC) y energía neta para lactancia $\left(E N_{L}\right)$ en seis secuencias de cultivo evaluadas durante los ciclos 2012-2013 y 2013-2014

\begin{tabular}{lcccccc}
\hline \multirow{2}{*}{ Secuencias de cultivo } & \multicolumn{2}{c}{$\mathrm{MS}\left(\mathrm{kg} \mathrm{m}^{-3}\right)$} & \multicolumn{2}{c}{$\mathrm{PC}\left(\mathrm{kg} \mathrm{m}^{-3}\right)$} & \multicolumn{2}{c}{ ENL $\left.(\mathrm{MJ} \mathrm{m})^{-3}\right)$} \\
\cline { 2 - 7 } & $2012-2013$ & $2013-2014$ & $2012-2013$ & $2013-2014$ & $2012-2013$ & $2013-2014$ \\
\hline Av-Mz-Mz & $1.65 \mathrm{~b}$ & $1.99 \mathrm{ab}$ & $0.135 \mathrm{~d}$ & $0.152 \mathrm{c}$ & $11.09 \mathrm{~b}$ & $13.72 \mathrm{a}$ \\
Av-Sr-Sr & $2.06 \mathrm{a}$ & $1.86 \mathrm{c}$ & $0.143 \mathrm{~d}$ & $0.119 \mathrm{~d}$ & $11.38 \mathrm{~b}$ & $10.25 \mathrm{c}$ \\
$\mathrm{Cn}-\mathrm{Tcl}-\mathrm{Mz}$ & $1.92 \mathrm{a}$ & $2.00 \mathrm{a}$ & $0.197 \mathrm{c}$ & $0.206 \mathrm{a}$ & $12.22 \mathrm{~b}$ & $13.05 \mathrm{ab}$ \\
$\mathrm{Cn}-\mathrm{Cb}-\mathrm{Mz}$ & $2.05 \mathrm{a}$ & $1.96 \mathrm{abc}$ & $0.204 \mathrm{bc}$ & $0.187 \mathrm{~b}$ & $13.64 \mathrm{a}$ & $12.59 \mathrm{~b}$ \\
Cn-Ctm-Mz & $1.90 \mathrm{a}$ & $1.89 \mathrm{bc}$ & $0.218 \mathrm{ab}$ & $0.215 \mathrm{a}$ & $11.88 \mathrm{~b}$ & $12.38 \mathrm{~b}$ \\
Cn-Tcl-Mp & $1.71 \mathrm{~b}$ & $1.49 \mathrm{~d}$ & $0.226 \mathrm{a}$ & $0.209 \mathrm{a}$ & $9.20 \mathrm{c}$ & $8.66 \mathrm{~d}$ \\
\hline
\end{tabular}

$\mathrm{Av}=$ avena; $\mathrm{Mz}=$ maíz; $\mathrm{Sr}=$ sorgo; $\mathrm{Cn}=$ canola; $\mathrm{Tcl}=$ triticale; $\mathrm{Cb}=$ cebada; $\mathrm{Ctm}=$ cártamo; $\mathrm{Mp}=$ mijo perla.

abcd Medias con la misma letra no son estadísticamente diferentes (DMS 0.05). 
La secuencia de cultivo con mijo perla registró una PA superior $(P<0.05)$ a los testigos en la producción de PC (58.0 a $67.4 \%$ ), sin embargo, en PA para MS, sus valores fueron iguales ( $P>0.05)$ a la secuencia con maíz e inferiores en $17.0 \%$ a la de la secuencia de cultivo con sorgo $(P<0.05)$. En la producción de $E N_{\mathrm{L}}$, la secuencia de cultivo con mijo perla mostró valores inferiores $(P<0.05)$ en $17.0 \%$ respecto a la secuencia con maíz y de $19.2 \%$ en relación a la secuencia con sorgo.

En el segundo ciclo, con un mayor rendimiento de MS en el maíz de la secuencia tradicional, el mejor testigo fue $\mathrm{Av}-\mathrm{Mz}-\mathrm{Mz}$, con valores de PA de $1.99 \mathrm{~kg}$ $\mathrm{m}^{-3}, 0.152 \mathrm{~kg} \mathrm{~m}^{-3}$ y $13.72 \mathrm{MJ} \mathrm{m}^{-3}$ en la producción de $\mathrm{MS}, \mathrm{PC}$ y $\mathrm{EN}_{\mathrm{L}}$, respectivamente. El testigo Av-SrSr registró menores valores $(P<0.05)$ de PA de MS, $P C$ y $E N_{L}$ en comparación al testigo con maíz, alcanzando disminuciones de 6.5, 21.7 y $25.3 \%$, respectivamente (Cuadro 5).

En todas las secuencias alternativas, el valor de PA para la producción de PC fue superior (23.0 a $41.4 \%$ ) a la obtenida por el mejor testigo Av-Mz-Mz $(P<0.05)$. Con excepción de la secuencia que incluyó mijo perla, el resto de las secuencias alternativas presentaron una PA mayor $(P<0.05)$ o similar $(P>0.05)$ al testigo en la producción de MS. En la producción de $\mathrm{EN}_{\mathrm{L}}$, sólo la secuencia $\mathrm{Cn}-\mathrm{Cb}-\mathrm{Mz}$ fue superior $(P<0.05)$ al patrón testigo $\mathrm{Av}-\mathrm{Mz}-\mathrm{Mz}$ en el primer ciclo; mientras que en el segundo, únicamente la secuencia $\mathrm{Cn}-\mathrm{Tcl}-\mathrm{Mz}$ presentó valores iguales al testigo $\mathrm{Av}-\mathrm{Mz}-\mathrm{Mz}$; las otras secuencias alternativas presentaron pérdidas $(P<0.05)$ entre 8.2 y $36.9 \%$.

Los valores de PA obtenidos por las secuencias convencionales en el presente estudio fueron similares o mayores a los observados en el testigo Av-Mz-Mz en otros estudios realizados en la región, en los cuales se observaron valores de 1.81 a $2.0 \mathrm{~kg}$ $\mathrm{m}^{-3}, 0.12$ a $0.18 \mathrm{~kg} \mathrm{~m}^{-3}$ y de 11.46 a $13.05 \mathrm{MJ} \mathrm{m}^{-3}$ para la producción de $M S$, PC y $E N_{L}$, respectivamente ${ }^{(2,13)}$. Estos resultados corroboran las ventajas de las secuencias alternativas observadas en el presente estudio, sobre todo en la producción de PC; los valores de PA obtenidos en este nutriente $\left(0.187\right.$ a $\left.0.226 \mathrm{~kg} \mathrm{~m}^{-3}\right)$ fueron iguales o superiores a los reportados en los estudios anteriores $^{(2,13)}$. Las ventajas observadas en PA en las secuencias alternativas fueron debido al menor requerimiento de agua y el mayor contenido de PC de las especies alternativas canola, cártamo, mijo perla, triticale y cebada respecto a las especies convencionales.

\section{CONCLUSIONES E IMPLICACIONES}

Las secuencias de cultivo alternativas con doble cosecha en otoño-invierno y la incorporación de especies forrajeras como canola, cártamo, triticale, cebada y mijo perla presentaron ventajas en potencial forrajero y productividad del agua respecto a las secuencias de cultivo tradicionales en la región. Las mejores secuencias alternativas incluyeron canola en otoño, cebada o triticale en invierno, y maíz en primavera, debido principalmente a los altos rendimientos de los cultivos de invierno. Estas secuencias alternativas, con menores valores de temperatura media y de evaporación potencial durante el ciclo, presentaron un menor requerimiento de agua, mayor rendimiento de proteína cruda y mayor productividad del agua en la producción de materia seca y proteína cruda. Sin embargo, también presentaron desventajas en los rendimientos de materia seca y energía neta para lactancia.

\section{AGRADECIMIENTOS}

Este estudio fue financiado por el Instituto Nacional de Investigaciones Forestales, Agrícolas y Pecuarias.

\section{LITERATURA CITADA}

1. Sanderson MG, Hemming DL, Betts RA. Regional temperature and precipitation changes under high-end $\left(\leq 4{ }^{\circ} \mathrm{C}\right)$ global warming. Philosophical Transactions of the Royal Society 2011;369:85-98.

2. Reta SDG, Figueroa VU, Faz CR, Núñez HG, Gaytán MA, Serrato CJS, Payán GJA. Sistemas de producción de forraje para incrementar la productividad del agua. Rev Fit Mex 2010;33 (Núm. Esp. 4):83-87.

3. Reta SDG, Serrato CJS, Figueroa VR, Cueto WJA, Berumen PS, Santamaría CJ. Cultivos alternativos con potencial de uso forrajero en la Comarca Lagunera. Libro Técnico núm. 3. INIFAP-CIRNOCCELALA. 2008.

4. Rajaram V, Nepolean T, Senthilvel S, Varshney RK, Vadez V, Srivastava RK, et al. Pearl millet (Pennisetum glaucum (L) R. Br.) consensus linkage map constructed using four RIL mapping 
populations and newly developed EST-SSRs. BMC Genomics 2013;14:159.

5. Santamaría CJ, Reta SDG, Faz CR, Orona CI. Reducción del rendimiento potencial en maíz forrajero en calendarios con tres y cuatro riegos. Terra Latinoamericana 2008;26:235-241.

6. Santamaría CJ, Reta SDG, Chávez GJFJ, Cueto WJA, Romero PRJI Caracterización del medio físico en relación a cultivos forrajeros alternativos para la Comarca Lagunera. Libro Técnico Núm. 2. INIFAP- CIRNOC-CELALA. 2006.

7. Harper FR, Berkenkamp B. Revised growth-stage key for Brassica campestris and B. napus. Canadian J Plant Sci 1975;55:657-658.

8. Goering HK, Van Soest PJ. Forage fiber analysis (apparatus, reagents, procedures, and some applications). Handbook 379. USDA-ARS, Washington, DC; 1970.

9. Bremner JM. Nitrogen-total. In: Sparks DL editor. Methods of soil analysis. Madison, WI: SSSA Book Ser 5; 1996:1085-1121.

10. NRC, National Research Council. Nutrient requirements of dairy cattle. Washington, DC: National Academy Press; 2001.

11. SAS Institute. SAS user's guide. Statistics, version 6.0. 4th. Ed. Cary, NC; 1990.

12. Cruz CJJ, Núñez HG, Faz CR, Reta SDG, Serrato MHA. Potencial forrajero y eficiencia de uso del agua de canola (Brassica napus L.) en comparación con cultivos tradicionales en el ciclo de invierno. AGROFAZ 2012;12:125-130.

13. Reta SDG, Figueroa VU, Serrato CJS, Quiroga GHM, Gaytán MA, Cueto WJA. Potencial forrajero y productividad del agua en patrones de cultivos alternativos. Rev Mex Cienc Pecu 2015;6:153-170.

14. Danieli PP, Primi R, Ronchi B, Ruggeri R, Rossini F, Del Puglia S, Cereti CF. The potential role of spineless safflower (Carthamus tinctorius L. var. inermis) as fodder crop in central Italy. Italian J Agr 2011;6:19-22.

15. Reta SDG, Serrato CJS, Gaytán MA, Quiroga GHM, Orozco HG, Payán GJA. Potencial forrajero del cártamo en respuesta al distanciamiento entre surcos en la Comarca Lagunera. AGROFAZ 2014;14:65-71.

16. Landau S, Friedman S, Brenner S, Bruckental I, Weinberg ZG, Ashbell G, et al. The value of safflower (Carthamus tinctorius) hay and silage grown under Mediterranean conditions as forage for dairy cattle. Livest Prod Sci 2004;88:263-271.

17. Kincaid RL, Johnson KA, Michal JJ, Huisman AC, Hulbert SH, Pan WL. Case study: production of silage containing biennial canola and peas for use as forage in a dairy ration. Profess Anim Scient 2012;28:120-124.

18. Núñez HG, Faz CR, Martínez RJG. Sistemas de producción de triple cosecha anual de forraje para la región Lagunera. AGROFAZ 2007;7:1-12. 